\title{
Etapas de la identidad para ser profesionista: evolución de las expectativas de los retos académicos a lo largo de la carrera
}

\author{
Silvia L. Olivares ${ }^{1 *}$, Nohemí Rivera ${ }^{2}$, Mildred V. López ${ }^{1}$ y Miriam L. Turrubiates ${ }^{1}$ \\ (1) Tecnologico de Monterrey, Escuela de Medicina y Ciencias de la Salud, Av. Morones Prieto 3000 Los Doctores, \\ 64710, Monterrey, México. (correo-e: solivares@tec.mx; mildredlopez@tec.mx; mturrubiates@tec.mx) \\ (2) Tecnologico de Monterrey, Departamento de Idiomas y Lenguaje, Av. Colegio Militar 4700, Nombre de Dios, 31300, \\ Chihuahua, México. (correo-e: nohemi_rivera@tec.mx)
}

* Autor a quien debe ir dirigida la correspondencia

Recibido Oct. 9, 2019; Aceptado Nov. 29, 2019; Versión final Ene. 8, 2020, Publicado Ago. 2020

\begin{abstract}
Resumen
El objetivo del presente estudio fue contrastar las etapas de identidad profesional de diferentes cohortes generacionales a partir de las expectativas, preferencias y logros que los estudiantes manifiestan hacia retos académicos. El método utilizado es cuantitativo, descriptivo y transeccional. Se diseñó un estudio de cohortes aplicando el Cuestionario de Etapas de Identidad Profesional en 1,583 alumnos de cuatro generaciones para medir las etapas de: autoconocimiento, relaciones de afinidad, conceptualización profesional y cambio consciente. Se observa mayor evolución de los alumnos en el último año de estudios en tres etapas. Los alumnos de primer año requieren reforzar su vocación e intereses mientras los mayores prefieren retos asociados a su profesión. En conclusión, el presente estudio muestra que hay diferencias en la identidad entre los alumnos de diversas cohortes de ingreso. Los estudiantes van cambiando las expectativas sobre ciertas vivencias académicas universitarias a medida que avanzan en su trayectoria educativa.
\end{abstract}

Palabras clave: identidad profesional; autoconocimiento; etapas de madurez; educación superior; retos

\section{Professional identity stages: evolution of expectations of academic challenges throughout the career program}

\begin{abstract}
The objective of the present study was to contrast the professional identity from different cohorts based on the expectations, preferences, and achievements that students rank on academic challenges. The method used was quantitative, descriptive, and cross-sectional. A comparative cohort method was selected. The Professional Identity Stages Questionnaire was applied to 1,583 students from four generations to measure the stages of self-knowledge, related relationships, professional conceptualization, and conscious change. Significant evolution was observed on the students in the last year in three of the stages. First-year students need to reinforce their vocation and interests, while older students prefer challenges associated with their profession. In conclusion, the present study showed that there were differences in identity between students from different cohorts. Students change their expectations on various academic experiences while they advance in their education.
\end{abstract}




\section{INTRODUCCIÓN}

La identidad es el entendimiento individual del desarrollo de la interacción que da significado a uno mismo y a los demás. Jarvis-Selinger et al., (2012) coinciden en que la formación de identidades es un proceso adaptativo que ocurre en dos niveles: 1) Individual: Involucra el desarrollo psicológico de la persona; y 2) Colectivo: Involucra la socialización de la persona en diversos roles en la comunidad. Jara y Mayor-Ruiz (2019) integran ambas visiones al considerar que la identidad es un proceso dinámico que se modifica con el tiempo dependiendo de las situaciones vividas por el sujeto y sus relaciones con los demás. Esto coincide con Scharager y Rodríguez (2019), quienes indican que es concebida como un proceso de diálogo y confrontación entre lo interno y externo; una interacción dinámica entre lo atribuido por otros y el intento de proyectar una identidad hacia el exterior, por lo cual requiere ser comprendida como una interacción entre personas y estructuras sociales.

Considerando un significado personal, la identidad es dinámica, múltiple, subjetiva y constituida a lo largo de la vida. De acuerdo a Monrouxe (2010) la identidad se construye dentro de los individuos y está en constante transformación. Esto implica una concepción dinámica donde el individuo posee múltiples facetas que conforman su identidad (Goldie, 2012). La identidad se describe como la apropiación del autoconocimiento y la autointerpretación de una narrativa autobiográfica que identifica signos y símbolos, mediante una mediación que recurre a los aspectos históricos y en ocasiones a características ficticias (Craig, 2005). Esto quiere decir que al ser un significado personal que surge de eventos e interpretaciones, fantasías o meditaciones, no es objetiva y puede ser reconstruida en la medida que el sujeto adquiere nuevas experiencias y significados (van Manen, 2003). Erikson y Erikson (1998) también concibe la identidad como el resultado subjetivo que surge de la experimentación del individuo consigo mismo durante toda su vida,

Desde una perspectiva social, el contexto y las relaciones constituyen la esencia de asociación y pertenencia. En ese sentido Beriain (1993), asegura que la definición de la identidad es selectiva y paradójica, ya que a nivel taxonómico la identidad se define en un borde o una frontera que representa a una empresa, entidad territorial, roles o partidos políticos. Es decir que la comunidad, la sociedad, o el colectivo imponen una identidad al grupo proporcionando al individuo de una identidad. Algunos autores la definen como la narración que el individuo hace sobre sí mismo, asegurando que se construye en la interacción simbólica con otros (Caballero, 2009). Esta se forma mediante un proceso dinámico de identificación en la que la persona se clasifica como individuo y a la vez como miembro de un colectivo.

Una de las múltiples facetas de la identidad de un individuo es la identidad profesional, la cual se forma en la universidad. La formación de la identidad profesional es el proceso por el cual un individuo se define a sí mismo como miembro de su profesión basado en la adquisición del conocimiento, habilidades, actitudes, valores y comportamientos requeridos (Slay y Smith, 2011). Considerando un modelo integrado entre lo individual y lo social, el presente trabajo centra la atención de la formación de la identidad profesional a través de los diversos años que transita como estudiante para convertirse en egresado. La educación superior ofrece experiencias para moldear esta faceta de identidad del individuo a través de su oferta educativa formal y extracurricular, la cual puede ser una oportunidad para potenciar su estructura de significados y la socialización con su entorno. La consideración de las implicaciones psicológicas del desarrollo es necesario para entender las complejidades de la formación de la identidad profesional. Las etapas de desarrollo de la identidad y el desarrollo moral, así como las experiencias únicas de cada individuo moldean la formación de dicha identidad. El proceso y los resultados son únicos para cada individuo (Holden et al., 2012).

Existen diferentes conceptualizaciones en cuanto a la formación de la identidad profesional. De acuerdo a lbarra (1999), a través del tiempo las personas requieren adaptarse a los nuevos roles que van adquiriendo y por lo tanto van modificando sus conductas de una forma adaptativa. Esto incluye modificar habilidades, actitudes y prácticas a partir de la observación, imitación o autoevaluación. Para encontrar sentido a la transición entre una identidad y otra, es importante considerar que además del "ser" existen "seres posibles", los cuales se derivan de las representaciones del ser en el pasado e incluyen representaciones del ser en el futuro. Estos posibles seres son un puente cognitivo entre el presente y el futuro, especificando cómo pueden cambiar los individuos de lo que son ahora a lo que serán. La meta de casi toda la investigación acerca del autoconcepto es relacionarlo con el comportamiento. La noción general es que si se desea cambiar el comportamiento se requiere cambiar ese autoconcepto. Sin embargo, es difícil establecer esa liga ya que el comportamiento de un individuo es muy variable y se cree que el autoconcepto es bastante estable. Sin embargo, parece que los autoconceptos pueden cambiar dramáticamente, dependiendo de la naturaleza de la situación social. El estudio de Markus y Nurius (1985) muestra que los individuos creen que pueden cambiar, incluso dramáticamente. Los seres posibles proveen un autoconcepto más complejo y variable, representando los deseos y temores del individuo e indicando lo que podrían realizar bajo ciertas condiciones.

Entonces, es importante saber que la transición entre una etapa de identidad y otra no es un proceso gradual de cambio, sino que está marcado por discontinuidades abruptas y es ocasionado por crisis emergentes (Erikson y Erikson, 1998). Estas crisis llegan porque se hacen evidentes las diferencias que existen entre lo que el individuo entiende de su rol profesional y su entendimiento de las experiencias y desafíos que está afrontando. 
Cuando se enfrenta a una crisis como esta, el individuo reevalúa la situación y empieza a incorporar nueva información y, en el mejor de los casos, desarrolla un nuevo entendimiento del mundo y de sí mismo (representando una nueva etapa de identidad) (Bejaard et al., 2004). Estas crisis sirven como momentos de cambio y representan un periodo de vulnerabilidad y agitación donde los valores o perspectivas viejas son evaluadas nuevamente (Erikson y Erikson, 1998). Cada experiencia y la evaluación individual de la experiencia se pueden ver como pequeñas contribuciones que moldean el todo (Holden, et al. 2012).

Entonces, la transición entre una etapa y otra implica dar un paso atrás al tiempo que los estudiantes luchan por incorporar las nuevas expectativas asociadas con la nueva identidad. Esto involucra tanto la formación de una nueva identidad como la destrucción de la vieja identidad. Al respecto, Heise (1977) afirmaba que las identidades van acompañadas de un sentimiento que sive como guía para interpretar y crear eventos. El comportamiento de un individuo es determinado por esfuerzos que confirman estos sentimientos, y si estos no pueden ser mantenidos, se debe seleccionar una nueva identidad. Scharager y Rodríguez (2019) hacen referencia a un estado ambiguo, en el que una persona tiene un estatus indeterminado en el que se encuentra atrapada en un espacio intermedio de transición. Asimismo, Wong y Trollope-Kumar (2014) afirman que los estudiantes entran a la escuela con identidades preexistentes (formadas por experiencias previas) y al avanzar en su entrenamiento, frecuentemente expresan ansiedad de perder aspectos valiosos de esas identidades, como volverse insensibles al sufrimiento. Entonces, la discontinuidad y la crisis son aspectos naturales y necesarios del proceso de desarrollo, y entenderlos es esencial si los educadores quieren apoyar a los estudiantes a manejar estas discontinuidades y navegar la transición de una identidad a otra (Jarvis et al., 2012).

De acuerdo a diversos autores, la formación de la identidad profesional se puede estructurar a través de etapas. Los autores en esta línea consideran que la formación de la identidad es un desarrollo progresivo incremental a través de una serie de fases (Hilton y Slotnick, 2005; Erikson y Erikson, 1998; Kegan, 1982). Estas etapas se caracterizan por ser distintas formas de entender tanto el ambiente que rodea al individuo, así como su rol en el mismo. De acuerdo a Forsythe (2005) el desarrollo moral es un componente clave en la formación de la identidad profesional. Kohlberg (1964) lo divide en 6 etapas (1. Obediencia y castigo, 2, Individualismo, 3, Expectativas interpersonales mutuas, 4. Sistema social y conciencia, 5. Contrato social y derechos individuales, 6 . Principios éticos fundamentales), de las cuales a partir de la etapa 2 que empieza una formación en educación superior asociada a la identidad profesional.

La etapa 2 (individualismo) se caracteriza por seguir las reglas sólo cuando es del interés inmediato de alguien; actuar para cumplir sus propios intereses y necesidades. Lo correcto es lo justo, lo que es un intercambio igual o un acuerdo. La perspectiva en esta etapa es individualista, consciente de que todos tienen sus propios intereses que perseguir, y estos pueden estar en conflicto, por lo que lo correcto es relativo. La etapa 3 (expectativas interpersonales mutuas) se caracterizan por vivir a la altura de lo que las personas cercanas esperan de ti. En esta etapa "ser bueno" es importante y significa tener buenos motivos, mostrar preocupación por los demás y mantener relaciones mutuas. El individuo es consciente de los sentimientos, acuerdos y expectativas compartidas, que ahora toman prioridad sobre los intereses individuales. Los individuos en la etapa 4 (sistema social y conciencia) se caracterizan por cumplir las tareas con las que se han comprometido. Lo correcto es contribuir a la sociedad, al grupo o a la institución y mantener esta estructura para evitar una ruptura en el sistema. Esta etapa se caracteriza por tomar el punto de vista del sistema, el cual define sus roles y reglas; además de considerar las relaciones individuales en términos de un lugar en el sistema (Kohlberg, 1964).

Los individuos en la etapa 5 (contrato social y derechos individuales) son conscientes de que las personas tienen una variedad de valores y opiniones, los cuales son relativos a un grupo. Sin embargo, algunos valores y derechos no relativos como la vida y la libertad, deben ser mantenidos en cualquier sociedad sin importar la opinión de la mayoría. La etapa se caracteriza por un sentido de obligación a la ley por el bien de todos y por la protección de los derechos de todas las personas. Existe una preocupación de que las leyes y responsabilidades se basen en la utilidad general "el mayor bien para el mayor número". Los individuos en la etapa 6 (principios éticos universales) siguen principios éticos elegidos por ellos mismos. La etapa se caracteriza por creer en los principios morales universales y por un sentido de compromiso personal hacia ellos. Zwet et al., (2010) coinciden en que los factores ambientales y conceptuales influyen fuertemente la manera en la que los estudiantes desarrollan la identidad profesional y aprenden a afrontar dilemas morales. Los individuos reconocen la naturaleza de la moralidad y el hecho de que las personas son fines en si mismos y deben ser tratadas como tal.

\section{OTROS ANTECEDENTES}

Hay diversos estudios que muestran el importante papel que juega la educación superior en la formación de la identidad profesional. Se esperaría que los alumnos de primer ingreso intenten adoptar los compartimentos de su profesión y en algún momento internalicen este rol hasta convertirse en un profesionista. Menéndez-Varela y Grigori-Giralt (2017) identifican una transición en la identidad profesional como una construcción durante el 
proceso formativo y la cual termina por interiorizarse en el trabajo. La figura 1 muestra cómo el candidato construye una idea sobre su futuro, el estudiante se forma en la profesión durante la universidad y sigue consolidando su identidad profesional a lo largo de la vida (figura 1).

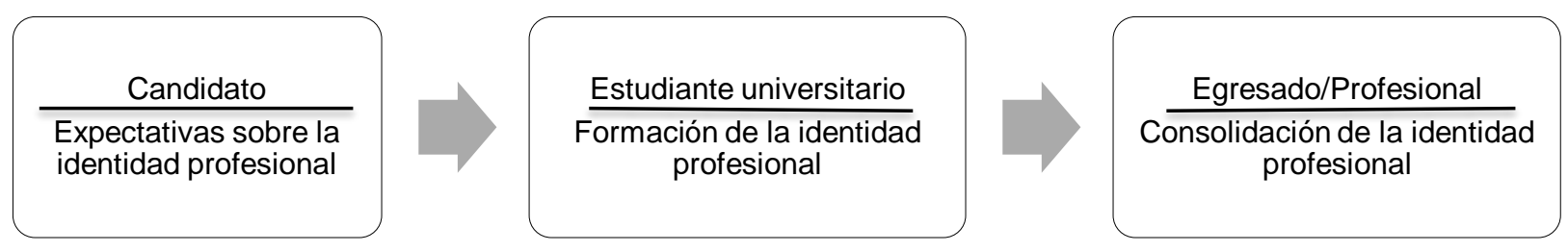

Fig.1: Interiorización de la identidad profesional

\section{El papel de la universidad para la formación de la identidad profesional}

La identidad profesional se construye de forma similar para las diferentes disciplinas. Por ejemplo, Hilton y Slotnick (2005) afirman que los estudiantes médicos experimentan la etapa de "pre-profesionalismo" antes de adquirir "madurez profesional" como resultado de su experiencia en la escuela y de la reflexión acerca de esa experiencia. Esto implica que el profesionalismo y la identidad profesional se dan como una progresión lineal a un estado de "madurez". En la universidad, los estudiantes co-construyen su identidad profesional a través de encuentros con sus colegas y mentores en una variedad de contextos, y a través de la reflexión de estas experiencias. Al tiempo que los estudiantes se mueven a través de su entrenamiento, desarrollan un entendimiento más sofisticado de su identidad profesional (Wong y Trollope-Kumar, 2014). Se puede decir que los descubrimientos más importantes son la naturaleza discontinua y negociada de la identidad profesional y las múltiples formas en que los individuos dan significado a los eventos e interacciones con otros en los ambientes académico y comunitario. En este sentido, Jarvis et al. (2012) sostienen que, aunque el entrenamiento de un médico pueda parecer una trayectoria lineal y fluida, hacia una meta, es en realidad un proceso de construir y abandonar una serie de identidades sucesivas, cada una con su propio conjunto de roles, perspectivas, derechos y responsabilidades.

Para los alumnos que se preparan en leyes, Gallardo (2019) cuestionó la pertinencia de la ética como elemento de la identidad profesional del abogado logrando una respuesta afirmativa. Su estudio indica que tal vínculo obedece a la naturaleza del derecho, al desarrollo histórico de la profesión y a la regulación que la profesión ha tenido. Si bien es evidente la necesidad de la ética en esta labor en particular, los mismos autores establecen que la ética es una competencia genérica que debe abordarse desde todas las profesiones. De forma similar Arias, Franco y Ceballos (2019) encontraron que los alumnos de ingeniería consideran que las actividades que más realizan en su carrera son: realizar cálculos, elaborar proyectos, realizar investigación y solucionar problemas, de las cuales estas últimas tres también podrían asociarse con otras carreras. Estas y otras competencias están consideradas en el modelo que proponen Olivares y Valdez (2017) para el desarrollo de competencias genéricas en cuatro dimensiones: individual, interpersonal, organizacional y sistémica, las cuales se asocian con la formación de la identidad profesional.

Debido a lo anterior, el alcance del presente trabajo es sobre la identidad como profesionista, sin importar la disciplina, considerando esta transformación a través de diversas etapas. Si bien las identidades ocupacionales en las universidades se han definido tradicionalmente a través de ámbitos estructurados como las disciplinas; en la actualidad el desdibujamiento de los límites institucionales han promovido la emergencia de un grupo cada vez más multiprofesional y diverso en la medida en que el trabajo se orienta por proyectos y contenidos integrados que cruzan fronteras funcionales y disciplinares (Scharager y Rodríguez, 2019). Este es el caso de la Semana i, donde se desarrollan habilidades útiles para la empleabilidad tales como; autoaprendizaje, pensamiento crítico, solución de problemas, curiosidad intelectual, innovación y trabajo colaborativo (Olivares et al., 2019). La Semana i se ha implementado desde el 2015 con el objetivo de promover y contribuir al desarrollo de competencias transversales para mas de 50,000 estudiantes universitarios de todas las disciplinas. De esta manera, es una estrategia inmersiva de aprendizaje activo mediante el Aprendizaje Basado en Retos, el cual se desarrolla en un contexto ajeno al aula, donde los participantes deben enfrentar un reto extraordinario que requiere un abordaje interdisciplinario, con la participación de alumnos, profesores y expertos externos (Olivares Olivares y Valdez-García, 2017).

De acuerdo al estudio de Olivares et al. (2019) los alumnos mencionan que además del aprendizaje general y la obtención de habilidades útiles para el ejercicio profesional, también encuentran un espacio para encontrarse consigo mismos y hacer vínculos con otros, lo cual los prepara para su vida personal y profesional. La oferta de actividades de Semana i es amplia. En el 2018 se ofrecieron un total de 1,295 actividades que se orientaron a: servicio comunitario, investigación, cultura, talleres disciplinares, certificaciones sobre habilidades técnicas entre otras. A continuación, se muestran algunos ejemplos. 


\section{Modelo dinámico de identidad profesional}

La identidad profesional es entonces la evolución dinámica a través de la cual el alumno se reconoce a sí mismo como persona que forma parte de grupos sociales y académicos para ejercer su rol dentro de contextos profesionales y de estar forma generar un cambio consciente. El enfoque del presente trabajo fusiona las ideas de cuatro modelos previamente mencionados de los autores Kohlberg (1964), Kegan (1982), Olivares Olivares y Valdez-García (2017) y Jara y Mayor Ruiz (2019) como se presenta en la tabla 1, para proponer cuatro etapas de un Modelo dinámico de identidad profesional (Figura 2).

Tabla 1: Comparación de autores que promueven la identidad por etapas o dimensiones

\begin{tabular}{|l|l|l|l|l|}
\hline & Kohlberg (1964) & Kegan (1982) & $\begin{array}{l}\text { Olivares Olivares y } \\
\text { Valdez-García (2017) }\end{array}$ & $\begin{array}{l}\text { Jara y Mayor Ruiz } \\
(2019)\end{array}$ \\
\hline Autoconocimiento & Individualismo & Imperial & Individual & $\begin{array}{l}\text { Reflexión del } \\
\text { proceso, Elementos } \\
\text { afectivos de la } \\
\text { identidad }\end{array}$ \\
\hline $\begin{array}{l}\text { Relaciones de } \\
\text { afinidad }\end{array}$ & $\begin{array}{l}\text { Expectativas interpersonales } \\
\text { mutuas }\end{array}$ & Interpersonal & Interpersonal & $\begin{array}{l}\text { Desempeño } \\
\text { profesional }\end{array}$ \\
\hline $\begin{array}{l}\text { Conceptualización } \\
\text { profesional }\end{array}$ & Sistema social y conciencia & Institucional & Organizacional & \\
\hline $\begin{array}{l}\text { Cambio } \\
\text { consciente }\end{array}$ & $\begin{array}{l}\text { Contrato social y derechos } \\
\text { individuales } \\
\text { Principios éticos universales }\end{array}$ & Interindividual & Sistémico & \\
\hline
\end{tabular}

A partir del marco teórico previamente presentado se propone un modelo dinámico de identidad profesional (Figura 2).

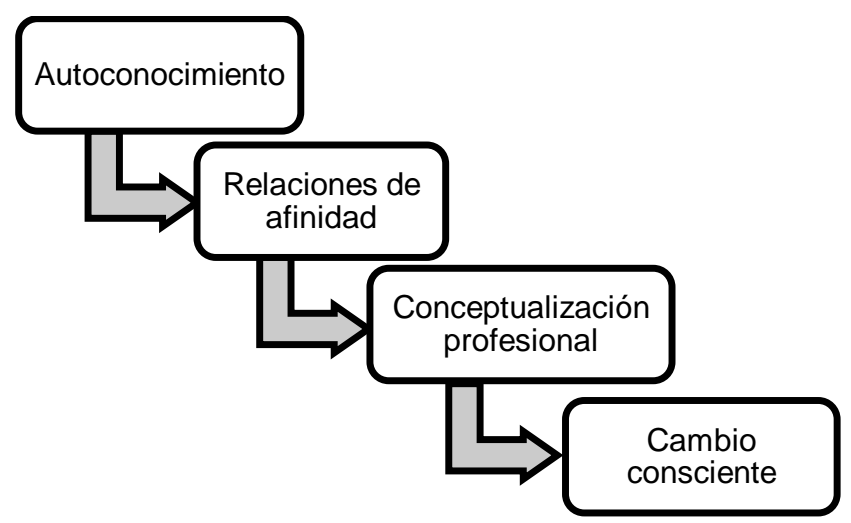

Fig.2: Modelo dinámico de identidad profesional

La etapa de Autoconocimiento implica que el individuo tenga claridad sobre sus valores y preferencias y tome decisiones alineadas a los mismos. Esto incluye la selección de una ruta profesional a partir del entendimiento profundo de sus capacidades y oportunidades de crecimiento, así como la planeación de una agenda de corto, mediano y largo plazo que le otorgue un sentido de independencia. Esto muestra similitudes con la etapa de individualismo de Kohlberg (1964) que se caracteriza por la búsqueda de cumplir con los propios intereses y necesidades. Asimismo, se relaciona con la etapa imperial de Kegan (1982) en la que los individuos adquieren un sentido de libertad e independencia al tomar el control de sus impulsos. Olivares Olivares y Valdez-García (2017) incluyen las competencias individuales las cuales son las cuentan con capa $\neg$ cidades cognitivas, de autodirección y técnicas que le permitan potenciar con-tinuamente su conocimiento. Para Jara y Mayor-Ruiz (2019) esta construcción de la identidad incluye una reflexión del proceso se asocia por una parte implica una reflexión del quehacer considerando aciertos y desaciertos de situaciones vividas por los propios protagonistas. También se asocia con los elementos afectivos de la identidad, dimensión que permite conocer los sentimientos y emociones con las que se va enfrentando el profesional.

Sin embargo, la libertad no debe considerarse como absoluta o ilimitada, pues deben considerarse el otro para no atropellar este mismo derecho en los demás. Por ejemplo Jarvis et al. (2012) establece que el entendimiento profundo de la profesión pudiera ser superficial cuando no se consideran estos elementos.

La etapa de Relaciones de afinidad se caracteriza por la construcción o ampliación de redes académicas, sociales y personales que sean afines a sus intereses. Esta interacción debe contribuir a compartir decisiones entre los miembros de dichas agrupaciones a nivel formal e informal. Esta etapa guarda una estrecha relación con la etapa de expectativas interpersonales mutuas (Kohlberg, 1964) en la que los sentimientos y expectativas compartidas toman prioridad sobre los intereses individuales. Además, se relaciona con la etapa interpersonal 
de Kegan (1982) en la que el individuo integra una pluralidad de voces y necesita de los otros para definirse a sí mismo y al mundo. De acuerdo a Olivares Olivares y Valdez-García (2017) las competencias interpersonales suponen relación de colaboración, las cuales incluyen el entendimiento profundo del otro. Para Jara y MayorRuiz (2019) la construcción de la identidad implica como parte del desempeño profesional donde se consideran las variables están enfocadas en las relaciones sociales y acciones dentro de su labor. En esta etapa los estudiantes internalizan las expectativas, comportamientos y valores de su profesión por lo que son muy sensibles a la percepción de los demás.

La etapa de Conceptualización profesional es cuando el individuo puede tomar decisiones asociadas con la profesión de su elección dentro de un contexto académico u organizacional. Es aceptado dentro de la comunidad de práctica como parte del equipo y participa activamente en la ejecución de tareas sencillas o complejas que sólo se delegan a los conocedores de la disciplina. Esta etapa es muy similar a la etapa de sistema social y conciencia (Kohlberg, 1964) que se caracteriza por un deseo de contribuir a la sociedad, al grupo o a la institución y mantener esta estructura. En esta etapa el individuo define sus roles y reglas con base en el punto de vista del sistema. También se puede ver una fuerte relación con la etapa institucional de Kegan (1982) en la que los individuos piensan en sí mismos en relación con sistemas más grandes en los que trabajan, construyendo un sistema personal de valores y procesos que usan para evaluar su rol dentro de la comunidad. De acuerdo a Olivares Olivares y Valdez-García (2017), las competencias organizacionales incluyen el desempeño dentro de los sistemas donde labora el individuo y donde puede establecer proyectos de mejora para la búsqueda de eficiencias, innovaciones y mejores experiencias para los clientes o usuarios. Para Jara y Mayor-Ruiz (2019) el desempeño profesional se asocia con las relaciones requeridas para la ejecución de proyectos y gestión de cada una de las labores profesionales. En esta etapa, las relaciones profesionales se han convertido en una parte natural de su mundo y han consolidado quienes son en relación con su comunidad de práctica.

Por último, la etapa de Cambio consciente se refiere a la persona que tiene la capacidad y flexibilidad para aportar valor liderando cambios para la sociedad. Acepta y valora las aportaciones de otros para construir mejores soluciones a las propuestas actuales de forma creativa y sustentable. Esta etapa se relaciona con las etapas 5 y 6 de Kohlberg (1964): Contrato social y derechos individuales y principios éticos universales, en las que el individuo es consciente de la diversidad de valores y opiniones. Olivares Olivares y ValdezGarcía (2017) establecen como impacto sistémico la capacidad para entender las variables complejas del entorno y actuar en consecuencia. Esta etapa se caracteriza por la búsqueda de proteger los derechos de todas las personas, siguiendo los principios morales universales. También existe una importante relación con la etapa interindividual de Kegan (1982) que se caracteriza por reconocer una pluralidad de seres y estar abiertos al conflicto emocional. En esta etapa, el individuo tiene una mayor capacidad para escuchar y buscar información, lo que lo hace ser flexible y estar siempre dispuesto a modificar su comportamiento.

\section{METODOLOGÍA}

El método utilizado es cuantitativo, descriptivo, transeccional y no experimental. Es cuantitativo porque se analizan los datos de autopercepción a través de una encuesta. De acuerdo con Valenzuela y Flores (2012) los estudios cuantitativos tienen como meta explicar y demostrar de manera objetiva la relación entre variables a través de instrumentos de recolección de datos analizados con herramientas estadísticas. En este sentido una encuesta en línea ofrece la ventaja de recoger información sin importar la ubicación del alumno. Es transeccional porque ocurre solamente en un momento del tiempo, en este caso durante septiembre del 2018. Es no experimental porque todos los estudiantes involucrados vivieron una experiencia retadora equivalente, es decir, no se comparan percepciones de alumnos ajenos a esta experiencia educativa (Valenzuela y Flores, 2012).

Se diseñó un estudio de cohortes, el cual describe el cambio mediante la selección de muestras distintas para cada momento temporal, formado por individuos que comparten un acontecimiento en común (Bisquerra, 2009). Para el presente estudio se considera la propuesta de Arnau, Anguera y Gómez (1990), la cual es un diseño de comparación de grupos donde se muestrean varias poblaciones que pertenecen al mismo grupo de ingreso a la universidad las cuales se valoran en un momento específico. En este sentido se tiene la finalidad en encontrar posibles diferencias entre datos obtenidos sobre las diversas cohortes.

Estos estudios estratifican las mediciones mediante matrices de dos variables donde una de ellas corresponde a los años (edad, acontecimiento en común) para contrastarla con la variable bajo estudio. Para el presente trabajo se contrasta los años de ingreso a la universidad con las etapas del modelo dinámico de identidad profesional.

La universidad donde se realizó el presente estudio tiene como misión formar líderes con espíritu emprendedor, sentido humano y competitivos internacionalmente. Es por esto que se ofrecen múltiples oportunidades educativas interprofesionales que buscan retar a los estudiantes para provocar el desarrollo de sus competencias y el reforzamiento de su identidad profesional a lo largo de sus estudios. En este sentido se vuelve relevante estudiar la contribución de la educación superior para facilitar la transición de sus estudiantes en las 
etapas de identidad profesional: Autoconocimiento, Relaciones de afinidad, Conceptualización profesional y Cambio consciente.

Para lograrlo, se han diseñado en el Tecnológico de Monterrey en México una amplia oferta de actividades retadoras en la que los alumnos solucionan problemas reales a partir de prácticas vivenciales, utilizando métodos o estrategias de inmersión durante una semana al año (Olivares et al, 2019). Los alumnos que ingresaron en el 2015 fueron los primeros en experimentar este tipo de vivencia académica y por tanto tienen diferente formación disciplinar y multidisciplinar en comparación con los que acaban de ingresar. Dado que el concepto de identidad profesional es dinámico y evolutivo, se esperaría que diferentes cohortes tuvieran diferentes opiniones estos conceptos, considerando que los alumnos próximos a graduarse pudieran tener más consolidada su identidad como profesionista, en comparación con los alumnos de primer año. El objetivo del presente estudio fue contrastar la identidad profesional de las diferentes cohortes a partir de las expectativas, preferencias y logros que los estudiantes manifiestan hacia los retos académicos que se plantean en esta semana de inmersión, mejor conocida como Semana i.

\section{Población y Muestra}

El estudio se realizó durante septiembre del 2018. La muestra corresponde a un total de 1,583 estudiantes que se estratifican por año de cohorte, quienes ingresaron a la institución a partir del 2015. Un conjunto de 281 corresponde al primer año (ingreso en 2018), 570 al segundo año (ingreso en 2017), 394 al tercer año (ingreso en 2016) y 338 a los próximos a graduarse (ingreso 2015). Los alumnos son una muestra aleatoria de una institución privada de educación superior a fin de estudiar su transición entre etapas de la identidad como profesionista. Debido a que los proyectos son multidisciplinarios, en el análisis no hay distinción entre disciplinas.

\section{Instrumento}

Como instrumento se adaptó el pre-test del Cuestionario de Autorreflexión de la Semana i de Olivares et al. (2019) el cual tiene un alpha de Cronbach de 0.95 en su versión original. Siguiendo los conceptos de la teoría de identidad profesional, se reclasificaron los reactivos para adaptarlas al Modelo dinámico de identidad profesional de la figura 2. En su versión original se contaba con 44 reactivos, los cuales se redujeron a 39 en la versión para este estudio. La versión adaptada se denominó "Cuestionario de etapas de identidad profesional" y cuenta con alpha de Cronbach de 0.97 , lo cual se considera confiable. En la tabla 2 se muestran ejemplos de los reactivos del cuestionario.

Tabla 2: Reactivos para cada una de las etapas

\begin{tabular}{|c|c|}
\hline Etapa de identidad & Reactivos \\
\hline Autoconocimiento & $\begin{array}{l}\text { Tengo claras mis metas de aprendizaje para esta Semana i. } \\
\text { Espero que la actividad seleccionada me rete intelectualmente. } \\
\text { Espero solucionar problemas relevantes para mi desarrollo profesional durante la Semana i. } \\
\text { Dedico tiempo a buscar información para aprender temas nuevos. } \\
\text { Comprendo las consecuencias de cada una de mis acciones. } \\
\text { Manejo la frustración ante las dificultades. }\end{array}$ \\
\hline $\begin{array}{l}\text { Relaciones de } \\
\text { afinidad }\end{array}$ & $\begin{array}{l}\text { Me comprometo a colaborar activamente con mis compañeros. } \\
\text { El diálogo entre los miembros de los equipos donde participo es cordial. } \\
\text { Motivo a otros a miembros de equipo, incluso en los momentos más críticos. } \\
\text { Los equipos en donde participo utilizan métodos efectivos para la toma de decisiones. }\end{array}$ \\
\hline $\begin{array}{l}\text { Conceptualización } \\
\text { profesional }\end{array}$ & $\begin{array}{l}\text { Me entusiasma aprender sobre la realidad de alguna organización. } \\
\text { Generalmente los miembros del equipo donde participo tienen claros sus roles y tareas. } \\
\text { Las organizaciones con las que he participado se han beneficiado de mis acciones. } \\
\text { Contribuyo a la sociedad con actividades altruistas al servicio de los demás. } \\
\text { Espero poder contribuir a alguna comunidad con mis acciones. }\end{array}$ \\
\hline Cambio consciente & $\begin{array}{l}\text { Dedico tiempo a reflexionar sobre mi aprendizaje y mis logros. } \\
\text { Me gustaría entender las problemáticas más relevantes de la sociedad. } \\
\text { Analizo una situación desde diferentes perspectivas antes de tomar una decisión. } \\
\text { Soy firme en mis valores, principios morales y convicciones. } \\
\text { Participo en actividades donde soy líder de equipos de trabajo. } \\
\text { Me interesa conocer la realidad de otros entornos y las oportunidades para aportar valor. } \\
\text { Espero adquirir aprendizaje de otras disciplinas para aplicarlo en mi carrera. }\end{array}$ \\
\hline
\end{tabular}




\section{Procedimiento de análisis de datos}

La escala de respuestas utilizada es de Likert de 1 a 5 donde 1 es totalmente en desacuerdo y 5 totalmente de acuerdo. Los resultados se analizan de forma descriptiva con la media en escala de 1 a 5 , estratificados por etapa de identidad profesional y para cada uno de las cohortes. Se utilizó la prueba de Kruskal Wallis (no paramétrica) al 95\% de confiabilidad para determinar si había diferencias significativas entre los mismos.

\section{RESULTADOS}

Los resultados del cuestionario se muestran en la tabla 3. Se observa que las respuestas del cuestionario, son estadísticamente diferentes a través de las cohortes en varias de las etapas de identidad. Los alumnos que están en a puntos de graduarse han cursado hasta 4 experiencias similares en el pasado y por lo tanto pueden tener mayores expectativas en cuanto a: Autoconocimiento, Conceptualización profesional y Cambio consciente.

Tabla 3: Resultados del cuestionario

\begin{tabular}{|l|l|l|l|l|l|}
\hline Etapa de identidad & $\begin{array}{l}\text { Primer año } \\
\text { (Ingresaron } \\
\text { 2018) }\end{array}$ & $\begin{array}{l}\text { Segundo año } \\
\text { (Ingresaron } \\
\text { 2017) }\end{array}$ & $\begin{array}{l}\text { Tercer año } \\
\text { (Ingresaron } \\
\text { 2016) }\end{array}$ & $\begin{array}{l}\text { Próximos a } \\
\text { graduarse } \\
\text { (Ingresaron 2015) }\end{array}$ & $\begin{array}{l}\text { p valor } \\
\text { Kruskal } \\
\text { Wallis }\end{array}$ \\
\hline Autoconocimiento & 4.37 & 4.45 & 4.43 & 4.49 & $0.00^{*}$ \\
\hline Relaciones de afinidad & 4.27 & 4.37 & 4.36 & 4.40 & 0.08 \\
\hline $\begin{array}{l}\text { Conceptualización } \\
\text { profesional }\end{array}$ & 4.33 & 4.41 & 4.37 & 4.43 & $0.02^{*}$ \\
\hline Cambio consciente & 4.31 & 4.39 & 4.37 & 4.41 & $0.02^{*}$ \\
\hline
\end{tabular}

Los resultados indican diferencia estadística significativa en la etapa de Autoconocimiento, es decir, cada una de las experiencias de Semana i manifiestan un cambio en el reconocimiento de los alumnos a sí mismos, las metas en su vida y el manejo de la frustración entre otras capacidades individuales. Entre las cohortes hay diferencias, las cuales son más evidentes entre la generación más avanzada y la de reciente ingreso. Según Kohlberg (1964) esta etapa se caracteriza por los intereses personales, que en este caso son las metas de aprendizaje de la actividad retadora, la búsqueda de nuevos conocimientos, así como la solución de problemas de utilidad para el desarrollo profesional. Kegan (1982) lo asocia con la libertad, que en este caso sería la selección voluntaria de cierta Semana i, según sus preferencias. Olivares Olivares y Valdez-García (2017) consideran que escritura, el uso de tecnología y la puntualidad son competencias individuales, las cuales aparentan potenciarse a medida que avanza el estudiante en los años universitarios. Jara y Mayor Ruiz (2019) dentro del concepto de identidad incluyen comprender los aciertos y desaciertos, así como los elementos afectivos, que en este caso los alumnos a punto de graduarse manifiestan niveles más altos de manejo de la frustración que los alumnos de años menos avanzados. Olivares et al. (2019) encontró que la Semana i, tiene impacto en las habilidades personales tales como el autoaprendizaje, lo cual coincide con el presente estudio.

La etapa de Conceptualización profesional también manifestó cambios. Los alumnos mayores tienen mayor deseo de hacer actividades asociadas con su profesión. La Semana i entonces, fortalece esta etapa y por lo tanto las actividades educativas para estos estudiantes deben estar mayormente alineadas a sus intereses profesionales, esto debido a que comienzan a internalizar las expectativas sociales, comportamientos y valores de la profesión (Kegan, 1982). Según Kohlberg (1964) esta etapa se caracteriza por un deseo de contribuir a la sociedad, lo cual se manifiesta con el énfasis de realizar actividades altruistas o apoyar a una comunidad. Para Olivares Olivares y Valdez-García (2017) y Jara y Mayor-Ruiz (2019) esta etapa se asocia con la ejecución de proyectos y la gestión de los recursos, que en este caso incluyen el beneficio a instituciones con un buen manejo del tiempo y el uso de métodos estructurados. Los alumnos próximos a graduarse tienen un promedio mayor en estas aseveraciones comparadas con los de primer ingreso.

Por último, también se manifiesta diferencia en la etapa de Cambio consciente, es decir, la universidad les convierte en líderes a través de las experiencias retadoras que ofrece de forma anual. En esta etapa los alumnos desean contribuir de forma más significativa con la sociedad a través de las actividades en las que participan. En esta etapa según Kohlberg (1964) el individuo es consciente de la diversidad de valores y opiniones, lo cual lo manifiestan declarando que son firmes hacia los mismos y se interesan de las problemáticas sociales. Olivares Olivares y Valdez-García (2017) establecen como impacto sistémico la realidad de los entornos, la innovación y el emprendimiento, los cuales se manifestaron con niveles más altos con los alumnos en etapas más avanzadas de sus programas. Olivares et al. (2019) encontró que la Semana i favorece la innovación, lo cual coincide con las expectativas sobre la creatividad y la búsqueda de métodos no tradicionales. En este sentido las prácticas multidisciplinarias en entornos de autenticidad permiten participar de forma activa en apoyo a la sociedad para un cambio consciente. 


\section{DISCUSIÓN}

Los alumnos a partir del segundo año muestran un avance considerable en cada una de las etapas, comparados con los alumnos de primer ingreso. Por el otro lado, los alumnos de tercer año muestran menores niveles en todas las etapas comparados con los alumnos de los dos años anteriores. Esto parece confirmar la teoría de Erikson y Erikson (1998) que afirma que las etapas de identidad profesional son discontinuas debido a crisis emergentes. Estas crisis llegan porque el estudiante percibe las diferencias entre lo que entiende de su rol profesional y las experiencias y desafíos que está afrontando. Estas crisis sirven como momentos de cambio y representan un periodo de vulnerabilidad donde las perspectivas viejas son evaluadas nuevamente (Erikson y Erikson, 1998). Entonces, la transición entre una etapa y otra puede implicar dar un paso atrás al tiempo que los estudiantes intentan incorporar las nuevas expectativas asociadas con la nueva identidad, destruyendo su vieja identidad.

La transición entre una etapa y otra no es un proceso de cambio gradual. Dado que los alumnos en años superiores están preparados para aportar ideas propias de su área disciplinar se hace relevante brindarles una oferta de retos académicos que les permita potenciar estas habilidades. En contraste, los alumnos de primer ingreso deben explorar sus intereses y prioridades dado que están en la etapa de Autoconocimiento y pueden participar en actividades que les permitan identificar sus propias fortalezas y debilidades. Esto coincide con un estudio de Bebeau y Monson (1985) quien utilizó un instrumento que mide las etapas de Kohlberg, y encontró que los estudiantes de primer año tienen diferente razonamiento para evaluar dilemas morales en comparación con los alumnos de tercer año. Así mismo coincide con el estudio de MenéndezVarela y Grigori-Giralt (2017) quienes encontraron que los alumnos de mayor progreso tenían mayor identidad sobre su profesión.

\section{CONCLUSIONES}

El presente estudio muestra que hay diferencias en la identidad entre los alumnos de diversas cohortes de ingreso. Como se estableció previamente, los estudiantes van avanzando por diferentes etapas de identidad a lo largo de su estancia por la universidad para consolidar su identidad una vez concluidos los estudios (Kohlberg, 1964; Kegan, 1982; Menéndez-Varela y Grigori-Giralt (2017). En esta ocasión una vivencia retadora de inmersión funcionó como experiencia educativa para evaluar los cambios entre las expectativas, preferencias y el sentido de logro a través de alumnos de diferentes generaciones. Los hallazgos de este estudio sugieren que los estudiantes van cambiando las expectativas sobre ciertas vivencias académicas universitarias a medida que avanzan en su trayectoria educativa, ya que se encuentran en una etapa diferente en la formación de su identidad profesional. En el presente trabajo se demuestra que los alumnos más avanzados también han evolucionado hacia un Cambio consciente profesional, por lo cual se recomienda seguir impulsando actividades de liderazgo y transformación en la educación superior.

\section{AGRADECIMIENTOS}

Se agradece a TecLabs del Tecnológico de Monterrey por el financiamiento del proyecto.

\section{REFERENCIAS}

Arias, W., Franco E., y Ceballos, K, Preferencias profesionales e identidad profesional en los estudiantes de ingenierías de una universidad privada de Arequipa, Propósitos y representaciones, 7(2), 160-195 (2019).

Arnau, J., Anguera M., y Gómez, J. Metodología de la investigación en ciencias del comportamiento, Universidad de Murcia, Secretariado de publicaciones, España (1990).

Bebeau, M.J., Rest J.R., Yamoor, C.M., Measuring dental students' ethical sensitivity, Journal of Dental Education, 49(4), 225-235 (1985).

Bejaard, D., Meijer, P.C., y Verloop, N, Reconsidering research on teachers' professional identity, Teaching and teacher education, 20(2), 107-128, (2004).

Beriain, J., De la sociedad industrial a la sociedad del riesgo, Revista Española de Investigaciones Sociológicas, 63(1),145162 (1993).

Bisquerra, R., Metodología de la investigación educativa, La Muralla, Madrid (2009).

Caballero, K, Construcción y desarrollo de la identidad profesional del profesorado universitario (Tesis Doctoral), Universidad de Granada, Granada, España (2009).

Craig, A.P., What is self? A Philosophy of Psychology. The Edwin Mellen Press, Lewiston (2005).

Erikson, E.H. y Erikson, J.M., The life cycle completed (extended version), WW Norton \& Company, New York, NY (1998). 
Forsythe, G.B., Identity development in professional education, Academic Medicine, 80(10), 112-117 (2005).

Gallardo, Y., ¿Es la ética jurídica un elemento de la identidad profesional del abogado? Los estudiantes opinan, Dikaion, 28(2) 284-309 (2019).

Goldie, J., The formation of professional identity in medical students: Considerations for educators, Medical Teacher, 34(1), 641-648 (2012).

Heise, D.R., Social action as the control of affect, Behavioral Science, 22(3), 163-177 (1977).

Hilton, S.R. y Slotnick, H.B., Proto-professionalism: How professionalisation occurs across the continuum of medical education, Medical Education, 39(1), 58-65 (2005).

Holden, M., Buck E., y otros tres autores. Professional identity formation in medical education: The convergence of multiple domains, HEC Forum, 24(4), 245-255 (2012).

Ibarra, H., Provisional selves: Experimenting with image and identity in professional adaptation, Administrative Science Quarterly, 44(4), 764-791 (1999).

Jara, C. y Mayor-Ruiz, C, Explorar la Construcción de la identidad docente en profesionales de la salud: diseño y validación de instrumento, Formación Universitaria, 12(1), 13-24 (2019).

Jarvis-Selinger, S., Pratt D.D., y Regehr, G., Competency is not enough: Integrating identity formation into the medical education discourse, Academic Medicine, 87(9), 1185-1190 (2012).

Kegan, R., The evolving self: Problem and process in human development, Harvard University Press, Cambridge, Mass: (1982).

Kohlberg, L., Development of Moral Character and Moral Ideology, In: Hofmann, M.L., Hofmann, L.W., Eds., Review of Child Development Research, pp. 381-431, Russell Sage Foundation, New York (1964).

Markus, H.y Nurius, P., Possible selves, American psychologist, 41(9), 954 (1986).

Menéndez-Varela, J.L., y Grigori-Giralt, E., La construcción de la identidad profesional de estudiantes universitarios de arte a través de proyectos de aprendizaje-servicio, Arte, individuo y Sociedad, 29(3), 417-443 (2017).

Monrouxe, L.V., Rees, C.E., y Hu, W., Differences in medical students' explicit discourses of professionalism: Acting, representing, becoming, Medical Education, 45(6), 585-602 (2011).

Olivares Olivares, S.L. y Valdez-García, J.E., Aprendizaje Centrado en el Paciente: Cuatro perspectivas para un abordaje integral, Editorial Médica Panamericana, México (2017).

Scharager, J., y Rodríguez, P., Identidad profesional de los administradores de la calidad en universidades chilenas: entre la invisibilización y la burocratización, Calidad en la Educación, 1(50), 254-283 (2019).

Slay, H.S. y Smith, D.A., Professional identity construction: Using narrative to understand the negotiation of professional and stigmatized cultural identities, Human Relations, 64(1), 85-107 (2011).

Valenzuela, J.R. y Flores, M., Fundamentos de investigación educativa, Vol. 2: El proceso de investigación educativa, Tecnológico de Monterrey, Escuela de Graduados en Educación: Monterrey, México (2012).

Van Manen, M., Investigación educativa y experiencia vivida: ciencia humana para una pedagogía de la acción y la sensibilidad. Idea Books SA, Barcelona (2003).

Wong, A. y Trollope-Kumar, K., Reflections: An inquiry into medical students' professional identity formation, Medical Education, 48(5), 489-501 (2014).

Zwet, J.V., Zwietering, P.J., y otros tres autores. Workplace learning from a socio-cultural perspective: Creating developmental space during the general practice clerkship, Advances in Health Sciences Education, 16(3), $359-373$ (2010). 